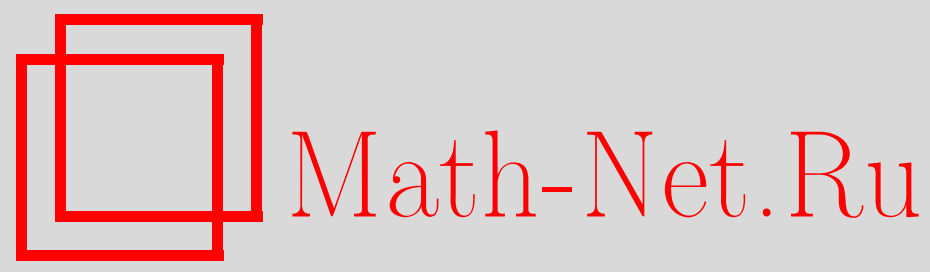

В. Л. Камынин, Об однозначной разрешимости обратной задачи для параболических уравнений с условием финального переопределения, Матем. заметки, 2003, том 73, выпуск 2, 217-227

DOI: https://doi.org/10.4213/mzm180

Использование Общероссийского математического портала Math-Net.Ru подразумевает, что вы прочитали и согласны с пользовательским соглашением http://www . mathnet.ru/rus/agreement

Параметры загрузки:

IP : 54.196 .121 .252

26 апреля 2023 г., 10:00:26

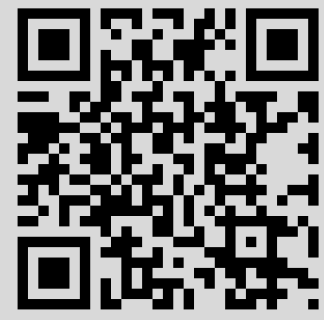


том 73 выпУСК 2 фЕВРАль 2003

\section{ОБ ОДНОЗНАЧНОЙ РАЗРЕШИМОСТИ ОБРАТНОЙ ЗАДАЧИ ДЛЯ ПАРАБОЛИЧЕСКИХ УРАВНЕНИЙ \\ С УСЛОВИЕМ ФИНАЛЬНОГО ПЕРЕОПРЕДЕЛЕНИЯ \\ В. Л. Камынин}

Изучается вопрос об однозначной разрешимости обратной задачи определения правой части для параболического уравнения со старшим коэффициентом, зависящим и от временной, и от пространственной переменной, при условии финального переопределения. Найдено два типа условий, достаточных для локальной разрешимости рассмотренной обратной задачи, а также доказана так назьваемая фредгольмова разрешшмость данной обратной задачи.

Библиограбоя: 10 названий.

1. Введение. В работе изучается вопрос об условиях однозначной разрешимости обратной задачи нахождения пары функций $\{u(t, x), f(x)\}$, удовлетворяюших уравнению

$$
\rho(t, x) u_{t}-\Delta u=f(x) g(t, x)+h(t, x), \quad(t, x) \in Q \equiv[0, T] \times \Omega,
$$

начальному условию

$$
u(0, x)=u_{0}(x), \quad x \in \Omega,
$$

граничному условию

$$
u(t, x)=0, \quad t \in[0, T], \quad x \in \partial \Omega,
$$

и условию переопределения

$$
u(T, x)=\varphi(x), \quad x \in \Omega,
$$

где $\Omega$ - ограниченная область в $\mathbb{R}^{n}$ с гладкой границей $\partial \Omega$.

Ранее обратныезадачи определения правой части параболического уравнения с условием финального переопределения изучались рядом авторов (см. [1]-[5] и др.). В [1], [2] изучался вопрос существования гладких решений соответствующей обратной задачи для параболических уравнений с гладкими, не зависящими от $t$ коэффициентами. Отдельно был рассмотрен случай $n=1$, где допускалась зависимость коэффициентов от $t$. В [3] рассматривалась обратная задача для уравнений, главная часть которых является оператором теплопроводности, но решения принадлежат классам Соболева. В [4]

Работа выполнена при финансовой поддержке Российского фонда фундаментальных исследований, грант № 00-01-00638. 
изучались обобщенные решения обратной задачи для параболического уравнения с коэффициентами, не зависящими от $t$.

В указанных работах были доказаны теоремы об однозначной разрешимости соответствующих обратных задач либо при условии определенной малости входящих в условие задачи данных (так называемая локальная разрешимость), либо при дополнительном условии, которое для уравнения (1.1) формулируется в виде

$$
g(t, x) \geqslant 0, \quad g_{t}(t, x) \geqslant 0, \quad(t, x) \in Q .
$$

Известные примеры показьвают, что те или иные дополнительные условия на входящие в задачу данные необходимы. Без указанных условий в [2], [4] для уравнений с гладкими коэффищиентами (в [2] допускалась зависимость коэффициентов также и от $t$ ) были доказаны теоремы о том, что из единственности решения соответствующей обратной задачи следует существование решения такой задачи (так называемая фредгольмова разрешимость обратной задачи).

В настоящей работе исследование однозначной разрешимости обратной задачи (1.1)-(1.4) проводится при существенно более слабых по сравнению с работами [1]-[5] требованиях на гладкость входящих в условие задачи функций. Получено несколько вариантов условий, обеспечивающих локальную разрешимость (в обобщенном смысле) задачи (1.1)-(1.4), а также теорема о фредгольмовой разрешимости рассматриваемой обратной задачи. Показано, что при $g(t, x)=$ const, $\rho(t, x)=$ const полученныев работе результаты включают в себя известные ранее.

Будем использовать следующие обозначения. Положим $Q_{t_{2}}^{t_{1}}=\left[t_{1}, t_{2}\right] \times \Omega, 0 \leqslant t_{1}<$ $t_{2} \leqslant T, Q_{t}^{0} \equiv Q_{t}, Q_{T}^{0} \equiv Q$. Пространства $L_{2}\left(Q_{t_{2}}^{t_{1}}\right), L_{2}(\Omega), W_{2}^{1,2}(Q), \stackrel{\circ}{W}_{2}^{1}(\Omega), C(0, T$; $\left.\stackrel{\circ}{W}_{2}^{1}(\Omega)\right), C\left(0, T ; L_{2}(\Omega)\right), W_{2}^{2}(\Omega)$ с соответствующими нормами будем понимать в общепринятом смысле (см., например, [6]).

Положим

$$
\begin{gathered}
\|v\| \equiv\|v\|_{L_{2}(\Omega)}, \quad v(x) \in L_{2}(\Omega) \\
\left\|v_{x}\right\|=\left(\int_{\Omega} \sum_{i=1}^{n} v_{x_{i}}^{2} d x\right)^{1 / 2}, \quad\left\|v_{x x}\right\|=\left(\int_{\Omega} \sum_{i, j=1}^{n} v_{x_{i} x_{j}}^{2} d x\right)^{1 / 2} .
\end{gathered}
$$

Через $\theta$ обозначим константу из неравенства Пуанкаре-Стеклова

$$
\|v\| \leqslant \theta\left\|v_{x}\right\|
$$

которое справедливо для любой $v(x) \in \stackrel{\circ}{W}_{2}^{1}(\Omega)$.

Мы будем использовать известное арифметическое неравенство

$$
|a b| \leqslant \frac{\varepsilon}{2} a^{2}+\frac{1}{2 \varepsilon} b^{2}, \quad \varepsilon>0, \quad a, b \in \mathbb{R}^{1} .
$$

Во всех дальнейших рассуждениях мы будем предполагать, что функции, входящие в исходные данные задачи, измеримы и удовлетворяют следуюшим условиям:

(A) $\Lambda_{1} \leqslant \rho(t, x) \leqslant \Lambda_{2},-K_{\rho, 1} \leqslant \rho_{t}(t, x) \leqslant K_{\rho, 2},(t, x) \in \bar{Q}$;

(B) $|g(t, x)| \leqslant K_{g},\left|g_{t}(t, x)\right| \leqslant K_{g}^{*},|g(T, x)| \geqslant g_{0}>0,(t, x) \in \bar{Q}$;

(C) $h(t, x) \in C\left(0, T ; L_{2}(\Omega)\right),\|h(t, \cdot)\| \leqslant K_{h}, h_{t}(t, x) \in L_{2}(Q),\left\|h_{t}\right\|_{L_{2}(Q)} \leqslant K_{h}$;

(D) $u_{0}(x) \in W_{2}^{2}(\Omega) \cap \stackrel{\circ}{W}_{2}^{1}(\Omega),\left\|u_{0}\right\|_{W_{2}^{2}(\Omega)} \leqslant M_{0}$;

(E) $\varphi(x) \in W_{2}^{2}(\Omega) \cap \stackrel{\circ}{W}_{2}^{1}(\Omega),\|\varphi\|_{W_{2}^{2}(\Omega)} \leqslant K_{\varphi}$. 
Здесь $\Lambda_{1}, \Lambda_{2}, K_{g}, g_{0}=$ const $>0, K_{\rho, 1}, K_{\rho, 2}, K_{g}^{*}, K_{h}, M_{0}, K_{\varphi}=$ const $\geqslant 0$.

Положим $K_{\rho}=\max \left\{K_{\rho, 1}, K_{\rho, 2}\right\} \geqslant 0$.

Ниже через $c$ (возможно, с индексом) будем обозначать любые положительные константы, зависящие от $\Lambda_{1}, \Lambda_{2}, K_{\rho}, K_{g}, g_{0}, K_{g}^{*}, K_{h}, M_{0}, K_{\varphi}, \theta$.

2. Сведение обратной задачи к операторному уравнению. Фредгольмова разрешимость обратной задачи.

ОПРЕДЕЛЕНИЕ 2.1. Обобщенным решением задачи (1.1)-(1.4) будем называть паpy функций $\{u(t, x), f(x)\}, u(t, x) \in C\left(0, T ; \stackrel{\circ}{2}_{2}^{1}(\Omega)\right), u_{t}, u_{x x} \in C\left(0, T ; L_{2}(\Omega)\right), f(x) \in$ $L_{2}(\Omega)$, удовлетворяющую уравнению (1.1) почти всюду в $Q$, причем функция $u(t, x)$ удовлетворяет условиям $(1.3),(1.4)$ в $\stackrel{(}{W}_{2}^{1}(\Omega)$.

Решение исходной обратной задачи будем искать в виде

$$
\{u(t, x), f(x)\}=\{z(t, x), f(x)\}+\{v(t, x), 0\},
$$

где $v(t, x)$ - решение в $Q$ прямой задачи

$$
\begin{aligned}
& \rho(t, x) v_{t}-\Delta v=h(t, x), \quad(t, x) \in Q, \\
& v(0, x)=u_{0}(x), \quad x \in \Omega, \\
& v(t, x)=0, \quad t \in[0, T], \quad x \in \partial \Omega,
\end{aligned}
$$

а пара $\{z(t, x), f(x)\}$ - решение в $Q$ обратной задачи

$$
\begin{aligned}
& \rho(t, x) z_{t}-\Delta z=f(x) g(t, x), \quad(t, x) \in Q, \\
& z(0, x)=0, \quad x \in \Omega, \\
& z(t, x)=0, \quad t \in[0, T], \quad x \in \partial \Omega, \\
& z(T, x)=\varphi(x)-v(T, x) \equiv \psi(x), \quad x \in \Omega .
\end{aligned}
$$

Обратимся к задаче (2.2)-(2.4).

ОПРЕДЕЛЕнИЕ 2.2. Энергетическим решением задачи (2.2)-(2.4) будем называть функцию $v(t, x) \in C\left(0, T ; L_{2}(\Omega)\right) \cap L_{2}\left(0, T ; \stackrel{\circ}{W}_{2}^{1}(\Omega)\right)$, удовлетворяющую соответствующему интегральному тождеству (см., например, $[6$, с. 158-164]).

В силу $[6$, c. 189] энергетическое решение задачи (2.2)-(2.4) существует и единственно. Однако в силу наших предположений это решение обладает дополнительной гладкостью.

Во-первых, в силу [7] $v(t, x) \in W_{2}^{1,2}(Q)$. Во-вторых, в силу теоремы 4.1, доказанной ниже в п. 4, функция $y(t, x) \equiv v_{t}(t, x)$ является энергетическим решением первой краевой задачи, получаемой из задачи $(2.2)-(2.4)$ путем формального дифференцирования ее по $t$ :

$$
\begin{aligned}
& \rho(t, x) y_{t}+\rho_{t}(t, x) y-\Delta y=h_{t}(t, x), \quad(t, x) \in Q, \\
& y(0, x)=\frac{\Delta u_{0}+h(0, x)}{\rho(0, x)}, \quad x \in \Omega, \\
& y(t, x)=0, \quad t \in[0, T], \quad x \in \partial \Omega .
\end{aligned}
$$


Из результатов работы [6] следует, что

$$
y(t, x) \equiv v_{t}(t, x) \in C\left(0, T ; L_{2}(\Omega)\right) \cap L_{2}\left(0, T ; \stackrel{\circ}{W}_{2}^{1}(\Omega)\right),
$$

причем

$$
\sup _{0 \leqslant t \leqslant T}\left\|v_{t}(t, \cdot)\right\|+\left\|v_{t x}\right\|_{L_{2}(Q)} \leqslant c_{1} .
$$

Учитьвая (2.12), нетрудно повторить рассуждения из [8, с. 116-117], в результате которых получается оценка

$$
\left\|v_{x x}(t, \cdot)\right\| \leqslant c_{2}
$$

Рассмотрим функцию $\psi(x)$ из соотношения (2.8). Из оценки $(2.13)$ и условия $(\mathrm{E})$ следует, что

$$
\psi \in \stackrel{\circ}{W}_{2}^{1}(\Omega) \cap W_{2}^{2}(\Omega),
$$

причем

$$
\|\psi\|_{W_{2}^{2}(\Omega)} \leqslant K_{\psi}
$$

где $K_{\psi}=$ const $>0$ зависит от $n, \Lambda_{1}, \Lambda_{2}, K_{\rho}, K_{\varphi}, M_{0}, K_{h}$.

ЗАмЕчАниЕ 2.1. В силу приведенных рассуждений и оценки (2.15) получаем, что изучение однозначной разрешимости задачи (1.1)-(1.4) эквивалентно исследованию однозначной разрешимости обратной задачи (2.5)-(2.8).

Выведем операторное уравнение относительно неизвестной функции $f(x)$.

Пусть $f(x) \in L_{2}(\Omega)$ произвольна. Тогда соотношения (2.5)-(2.7) представляют собой первую краевую задачу для нахождения функции $z(t, x)$. Повторяя рассуждения, проведенные выше для задачи (2.2)-(2.4), получим, что для любой $f(x) \in L_{2}(\Omega)$ существует единственное решение $z(t, x)$ задачи $(2.5)-(2.7)$, причем

$$
\begin{gathered}
z(t, x) \in C\left(0, T ; \stackrel{\circ}{2}_{2}^{1}(\Omega)\right), \\
z_{t} \in C\left(0, T ; L_{2}(\Omega)\right) \cap L_{2}\left(0, T ; \stackrel{\circ}{2}_{2}^{1}(\Omega)\right), \quad z_{x x} \in C\left(0, T ; L_{2}(\Omega)\right) .
\end{gathered}
$$

Полагая в (2.5) $t=T$ и учитывая (2.8), приходим к соотношению

$$
\rho(T, x) z_{t}(T, x)-\Delta \psi=f(x) g(T, x),
$$

откуда

$$
f(x)=\frac{\rho(T, x)}{g(T, x)} z_{t}(T, x)-\frac{\Delta \psi}{g(T, x)} .
$$

Введем линейный оператор $B: L_{2}(\Omega) \rightarrow L_{2}(\Omega)$ по формуле

$$
B(f)=\frac{\rho(T, x)}{g(T, x)} z_{t}(T, x),
$$

где $z(t, x)$ - решение прямой задачи (2.5)-(2.7) при заданной функции $f(x)$ в правой части уравнения (2.5). В силу (2.19) соотношение (2.18) можно записать в виде операторного уравнения

$$
f=B(f)-\frac{\Delta \psi}{g(T, x)} .
$$


ЛЕмма 2.1. Пусть выполнены условия (А)-(Е). Тогда для того чтобы пара $\{z, f\}$ была обобщенным решением задачи (2.5)-(2.8), необходимо и достаточно, чтобы эта пара удовлетворяла соотношениям (2.5)-(2.7), (2.20).

ДокАЗАТЕльство. Необходимость доказана вьше при вьводе соотношения (2.20).

Докажем достаточность. Итак, пусть $f^{*}(x) \in L_{2}(\Omega)$ является решением уравнения (2.20). Рассмотрим функцию $z^{*}(t, x)$ как единственное решение прямой задачи (2.5)-(2.7) с выбранной функцией $f(x) \equiv f^{*}(x)$ в правой части уравнения (2.5). Как и вьше, получим, что $z^{*}(t, x)$ удовлетворяет (2.16). Положим

$$
\psi^{*}(x)=z^{*}(T, x) \in W_{2}^{2}(\Omega) \cap \stackrel{\circ}{W}_{2}^{1}(\Omega) .
$$

Тогда, очевидно,

$$
\rho(T, x) z_{t}^{*}(T, x)-\Delta \psi^{*}=f^{*}(x) g(t, x) .
$$

$\mathrm{C}$ другой стороны, $f^{*}(x)$ является решением уравнения $(2.20)$ и в силу определения оператора $B$ имеем, что $f^{*}(x)$ удовлетворяет соотношению

$$
\rho(T, x) z_{t}^{*}(T, x)-\Delta \psi=f^{*}(x) g(t, x) .
$$

Сравнивая его с (2.22), получаем, что

$$
\Delta \psi^{*}(x)=\Delta \psi(x), \quad x \in \Omega .
$$

Из последнего соотношения с учетом (2.14) и (2.21) вытекает, что $\psi^{*}(x)=\psi(x)$ в $\Omega$, а следовательно, пара $\left\{z^{*}(t, x), f^{*}(x)\right\}$ является решением обратной задачи $(2.5)-(2.8)$.

Лемма доказана.

Лемма 2.2. Пусть выполнены условия (А)-(Е). Тогда оператор В, введенный соотношением (2.19), является вполне непрерывным как оператор из $L_{2}(\Omega)$ в $L_{2}(\Omega)$.

ДокАЗАТЕЛЬСТво. Пусть $f(x) \in L_{2}(\Omega)$. Пусть $z(t, x)$ - решение прямой задачи $(2.5)-(2.7)$. Положим $w(t, x)=z_{t}(t, x)$. Тогда в силу $(2.16)$

$$
w(t, x) \in C\left(0, T ; L_{2}(\Omega)\right) \cap L_{2}\left(0, T ; \stackrel{\circ}{W}_{2}^{1}(\Omega)\right),
$$

причем из доказанной ниже теоремы 4.1 вытекает, что $w(t, x)$ является в $Q$ энергетическим решением первой краевой задачи

$$
\begin{aligned}
& (\rho w)_{t}-\Delta w=f(x) g_{t}(t, x), \\
& w(0, x)=\frac{f(x) g(0, x)}{\rho(0, x)}, \quad x \in \Omega, \\
& w(t, x)=0, \quad t \in[0, T], \quad x \in \partial \Omega,
\end{aligned}
$$

которая получается из задачи (2.5)-(2.7) путем формального дифференцирования по $t$.

Из (2.23) следует, что для почти всех $t \in(0, T]$ вьполнено $w_{x}(t, x) \in L_{2}(\Omega)$. Выберем и фиксируем $\varepsilon \in(0, T)$ так, чтобы $w_{x}(\varepsilon, \cdot) \in L_{2}(\Omega)$. Рассмотрим в $Q_{T}^{\varepsilon}$ первую краевую задачу

$$
\begin{aligned}
& (\rho y)_{t}-\Delta y=f(x) g_{t}(t, x), \\
& y(\varepsilon, x)=w(\varepsilon, x), \quad x \in \Omega, \\
& y(t, x)=0, \quad t \in[\varepsilon, T], \quad x \in \partial \Omega .
\end{aligned}
$$


В силу [6, с. 202-206] такая задача имеет единственное энергетическое решение $y(t, x)$, причем, пользуясь методами работ [6, с. 212] и [9], нетрудно показать, что

$$
y(t, x) \in C\left(\varepsilon, T ; \stackrel{\circ}{W}_{2}^{1}(\Omega)\right) \cap W_{2}^{1,2}\left(Q_{T}^{\varepsilon}\right) .
$$

Очевидно, что $w(t, x)$ также является энергетическим решением задачи $(2.27)-(2.29)$ (см. соотношение $(2.23)$ ). Следовательно, $w(t, x)=y(t, x)$ в $Q_{T}^{\varepsilon}$, и из (2.30) вытекает тогда, что

$$
w_{x}(t, x) \in C\left(\varepsilon, T ; L_{2}(\Omega)\right) .
$$

Из теоремы о среднем в силу (2.31) вытекает существование $\tau^{*} \in[\varepsilon, T]$ такого, что

$$
\int_{\varepsilon}^{T} \int_{\Omega}\left|w_{x}(t, x)\right|^{2} d x d t=(T-\varepsilon)\left\|w_{x}\left(\tau^{*}, \cdot\right)\right\|^{2}
$$

Перепишем уравнение (2.24) в виде

$$
-w_{t}+\frac{1}{\rho(t, x)} \Delta w=\frac{\rho_{t}(t, x)}{\rho(t, x)} w-\frac{g_{t}(t, x)}{\rho(t, x)} f
$$

умножим его на $\Delta w$ и проинтегрируем по цилиндру $Q_{T}^{\tau^{*}}$. После несложных преобразований с применением неравенства (1.7) получим соотношение

$$
\frac{1}{2}\left\|w_{x}(T, \cdot)\right\|^{2}+\frac{1}{2 \Lambda_{2}}\|\Delta w\|_{L_{2}\left(Q_{T}^{\tau^{*}}\right)}^{2} \leqslant c_{1} T\|f\|^{2}+c_{2}\|w\|_{L_{2}\left(Q_{T}^{\tau^{*}}\right)}^{2}+\frac{1}{2}\left\|w_{x}\left(\tau^{*}, \cdot\right)\right\|^{2},
$$

из которого после использования (2.32) приходим к оценке

$$
\frac{1}{2}\left\|w_{x}(T, \cdot)\right\|^{2} \leqslant c_{1} T\|f\|^{2}+c_{2}\|w\|_{L_{2}\left(Q_{T}^{\tau^{*}}\right)}^{2}+\frac{1}{2(T-\varepsilon)}\left\|w_{x}\right\|_{L_{2}\left(Q_{T}^{\tau^{*}}\right)}^{2} .
$$

Умножим теперь уравнение $(2.24)$ на $w(t, x)$ и проинтегрируем по цилиндру $Q_{t}$. Тогда с учетом (2.26) после интегрирования по частям придем к неравенству

$$
\frac{\Lambda_{1}}{2}\|w(t, \cdot)\|^{2}+\left\|w_{x}\right\|_{L_{2}\left(Q_{t}\right)}^{2} \leqslant c_{3}\|f\|^{2}+K_{\rho}\|w\|_{L_{2}\left(Q_{t}\right)}^{2}+K_{g}^{*} \sqrt{t}\|f\| \cdot\|w\|_{L_{2}\left(Q_{t}\right)} .
$$

Из последнего неравенства вытекает, что

$$
\begin{aligned}
\frac{\Lambda_{1}}{2} \sup _{0 \leqslant \tau \leqslant t}\|w(\tau, \cdot)\|^{2}+\left\|w_{x}\right\|_{L_{2}\left(Q_{t}\right)}^{2} & \leqslant\left(K_{\rho}+1\right)\|w\|_{L_{2}\left(Q_{t}\right)}^{2}+\left(c_{3}+t K_{g}^{* 2}\right)\|f\|^{2} \\
& \leqslant\left(K_{\rho}+1\right) t \cdot \sup _{0 \leqslant \tau \leqslant t}\|w(\tau, \cdot)\|^{2}+c_{4}(T)\|f\|^{2} .
\end{aligned}
$$

Пусть $t \equiv t_{0}$ таково, что

$$
\left(K_{\rho}+1\right) t<\frac{\Lambda_{1}}{4}
$$

Тогда из (2.34) следует оценка

$$
\frac{\Lambda_{1}}{4} \sup _{0 \leqslant \tau \leqslant t_{0}}\|w(\tau, \cdot)\|^{2}+\left\|w_{x}\right\|_{L_{2}\left(Q_{t_{0}}\right)}^{2} \leqslant c_{4}(T)\|f\|^{2} .
$$


Выбор $t_{0}$ в $(2.35)$ не зависит от начального условия $(2.26)$, поэтому за конечное число шагов мы получим оценку

$$
\sup _{0 \leqslant t \leqslant T}\|w(t, \cdot)\|^{2}+\left\|w_{x}\right\|_{L_{2}(Q)}^{2} \leqslant c_{5}(T)\|f\|^{2} .
$$

Подставляя (2.36) в (2.33), получаем, что

$$
\left\|w_{x}(T, \cdot)\right\|^{2} \leqslant c_{6}(T, \varepsilon)\|f\|^{2} .
$$

Поскольку $\varepsilon \in(0, T)$ было фиксировано, то из (2.37) следует оценка

$$
\left\|z_{t x}(T, \cdot)\right\|^{2} \equiv\left\|w_{x}(T, \cdot)\right\|^{2} \leqslant c^{*}(T)\|f\|^{2} .
$$

Из оценки (2.38), определения оператора $B$ и компактности вложения пространства $\stackrel{\circ}{W}_{2}^{1}(\Omega)$ в $L_{2}(\Omega)$ следует, что оператор $B$ вполне непрерьвен как оператор из $L_{2}(\Omega)$ в $L_{2}(\Omega)$.

Лемма 2.2 доказана.

ТЕОрема 2.1. При сделанных предположениях (A)-(Е) обратная задача (1.1)(1.4) является фредгольмово разрешимой, т.е. из единственности решения обратной задачи (1.1)-(1.4) следует существование решения этой задачи.

ДокАЗАтЕльСтво. В силу замечания 2.1 теорему достаточно доказать для задачи (2.5)-(2.8). В силу леммы 2.2 оператор $B$ является вполне непрерьвным. Поэтому утверждение теоремы для задачи (2.5)-(2.8) следует из известных результатов функционального анализа $[10$, с. 472$]$ и леммы 2.1 .

3. Локальная разрешимость обратной задачи. Дадим два типа условий, достаточных для однозначной разрешимости обратной задачи (1.1)-(1.4).

ТЕОРема 3.1. Пусть выполнены условия (A)-(Е). Предположим дополнительно, что

$$
\begin{gathered}
\gamma \equiv \frac{1}{\theta^{2}}-\frac{K_{\rho, 1}}{2}>0, \\
\frac{g^{2}(T, x)}{\rho(T, x)}-\frac{g^{2}(0, x)}{\rho(0, x)} \geqslant \mu_{0}>0, \quad x \in \Omega, \\
T<\frac{\left(2-K_{\rho, 1}\right) \mu_{0}}{2 \theta^{2} K_{g}^{* 2}} .
\end{gathered}
$$

Тогда существует обобщенное решение задачи (1.1)-(1.4) и оно единственно.

ДокАЗАтЕльство. І. Единственность решения. Покажем, что решение задачи (1.1)-(1.4) единственно. Пусть $\{u(t, x), f(x)\}$ - решение задачи (1.1)-(1.4) при $h(t, x)=\varphi(x)=u_{0}(x) \equiv 0$. Тогда функция $w(t, x) \equiv u_{t}(t, x)$ является решением задачи $(2.24)-(2.26)$.

Умножим уравнение $(2.24)$ на $w(t, x)$ и проинтегрируем по $Q$. Учитывая условие (3.1), после несложных преобразований с использованием неравенства (1.7) приходим к оценке

$$
\int_{\Omega} \rho(T, x) w^{2}(T, x) d x-\int_{\Omega} \rho(0, x) w^{2}(0, x) d x+\gamma \int_{Q} w^{2} d x d t \leqslant \frac{2 \theta^{2} K_{g}^{* 2}}{2-K_{\rho, 1} \theta^{2}} T\|f\|^{2} .
$$


Из уравнения (1.1) при $t=T$ с учетом (1.4) и условия $\varphi(x) \equiv 0$ имеем

$$
f(x)=\frac{\rho(T, x)}{g(T, x)} u_{t}(T, x) \equiv \frac{\rho(T, x)}{g(T, x)} w(T, x) .
$$

Подставляя соотношения (3.5) и (2.25) в (3.4), приходим к оценке

$$
\int_{\Omega}\left[\frac{g^{2}(T, x)}{\rho(T, x)}-\frac{g^{2}(0, x)}{\rho(0, x)}\right] f^{2}(x) d x+\gamma\|w\|_{L_{2}(Q)}^{2} \leqslant \frac{2 \theta^{2} K_{g}^{* 2}}{2-K_{\rho, 1} \theta^{2}} T\|f\|^{2} .
$$

В силу (3.2) имеем отсюда

$$
\mu_{0}\|f\|^{2}+\gamma\|w\|_{L_{2}(Q)}^{2} \leqslant \frac{2 \theta^{2} K_{g}^{* 2}}{2-K_{\rho, 1} \theta^{2}} T\|f\|^{2},
$$

что в силу (3.3) влечет $f(x) \equiv 0$ в $\Omega, w \equiv u_{t} \equiv 0$ в $Q ;$ но тогда, очевидно, и $u(t, x) \equiv 0$ в $Q$.

Таким образом, единственность решения задачи (1.1)-(1.4) доказана.

II. Сушествование решения обратной задачи (1.1)-(1.4) есть следствие его единственности и утверждения теоремы 2.1.

Теорема 3.1 доказана.

ЗАмЕчАниЕ 3.1. 1) Условие (3.1) теоремы 3.1 заведомо выполнено, если $\rho_{t}(t, x) \geqslant 0$ в $Q$.

2) Условие (3.2) теоремы 3.1 заведомо вьполнено, если $g(0, x) \equiv 0, x \in \Omega$.

ТЕОРема 3.2. Пусть выполнены условия (A)-(Е). Предположим дополнительно, что

$$
\begin{gathered}
\nu \equiv \frac{1}{\Lambda_{2} \theta^{2}}-\frac{K_{\rho, 1}}{\Lambda_{1}}>0 \\
\frac{\Lambda_{2}^{2}}{\Lambda_{1} g_{0}^{2}}\left[\left(\frac{K_{g}^{2}}{\Lambda_{1}}-\frac{\theta^{2} K_{g}^{* 2}}{\nu}\right) e^{-\nu T}+\frac{\theta^{2} K_{g}^{* 2}}{\nu}\right]<1 .
\end{gathered}
$$

Тогда обратная задача (1.1)-(1.4) имеет решение и оно единственно.

ДокАЗАТЕЛьСтво. І. Сушествование решения задачи (1.1)-(1.4). Докажем прежде всего, что при вьполнении условий (3.6), (3.7) оператор $B$ в (2.19) является сжимающим оператором из $L_{2}(\Omega)$ в $L_{2}(\Omega)$.

Умножим уравнение $(2.24)$ на $w(t, x)$ и проинтегрируем по $\Omega$. После несложных преобразований с применением неравенства (1.7) приходим к оценке

$$
\frac{d}{d t} \int_{\Omega} \rho w^{2} d x+\left(\frac{1}{\Lambda_{2} \theta^{2}}-\frac{K_{\rho, 1}}{\Lambda_{1}}\right) \int_{\Omega} \rho w^{2} d x \leqslant \theta^{2} K_{g}^{* 2}\|f\|^{2} .
$$

Заметим, что в силу (3.6) множитель $1 /\left(\Lambda_{2} \theta^{2}\right)-K_{\rho, 1} / \Lambda_{1} \equiv \nu>0$, поэтому, применяя к (3.8) известную лемму Гронуолла (см., например, [6, с. 112]) и учитьвая условие (2.25), приходим к оценке

$$
\int_{\Omega} \rho(T, x) w^{2}(T, x) d x \leqslant \int_{\Omega} \frac{g^{2}(0, x)}{\rho(0, x)} f^{2}(x) d x \cdot e^{-\nu T}+\frac{\theta^{2} K_{g}^{* 2}}{\nu}\left(1-e^{-\nu T}\right)\|f\|^{2} .
$$


Следовательно,

$$
\|B f\|^{2} \equiv\left\|\frac{\rho(T, \cdot)}{g(T, \cdot)} w(T, \cdot)\right\|^{2} \leqslant \frac{\Lambda_{2}^{2}}{\Lambda_{1} g_{0}^{2}}\left[\left(\frac{K_{g}^{2}}{\Lambda_{1}}-\frac{\theta^{2} K_{g}^{* 2}}{\nu}\right) e^{-\nu T}+\frac{\theta^{2} K_{g}^{* 2}}{\nu}\right]\|f\|^{2} .
$$

Из (3.9) в силу (3.7) вытекает сжимаемость оператора $B$. Следовательно, уравнение (2.20) однозначно разрешимо.

Пусть $f(x) \in L_{2}(\Omega)$ является его решением. Подставляя данную $f(x)$ в правую часть уравнения (2.5), найдем функцию $z(t, x)$ как решение прямой задачи (2.5)-(2.7). Тогда в силу леммы 2.1 пара $\{z, f\}$ будет обобщенным решением обратной задачи $(2.5)-(2.8)$, что с учетом замечания 2.1 влечет за собой разрешимость обратной задачи (1.1)-(1.4).

II. Единственность решения. Предположим, что существует два различных решения $\left\{u_{1}, f_{1}\right\}$ и $\left\{u_{2}, f_{2}\right\}$ обратной задачи (1.1)-(1.4). Тогда в силу леммы 2.1 существует два различных решения $\left\{z_{1}, f_{1}\right\}$ и $\left\{z_{2}, f_{2}\right\}$ обратной задачи (2.5)-(2.8). При этом обязательно

$$
f_{1}(x) \not \equiv f_{2}(x),
$$

поскольку иначе в силу единственности решения прямой задачи мы бы имели $z_{1}(t, x) \equiv$ $z_{2}(t, x)$. Однако условие (3.10) противоречит сжимаемости оператора $B$, поскольку обе функции $f_{1}(x)$ и $f_{2}(x)$ являются решениями одного и того же операторного уравнения (2.20).

Теорема 3.2 доказана.

ЗАмечАниЕ 3.2. Если $g(t, x) \equiv$ const, $\rho(t, x) \equiv$ const в $Q$, то условия $(3.6),(3.7)$ всегда выполнены, и обратная задача (1.1)-(1.4) имеет единственное решение, что соответствует известньм ранее результатам.

ЗАмЕчАНИЕ 3.3. Условие (3.6) заведомо вьполнено, если $\rho_{t}(t, x) \geqslant 0$ в $Q$.

ЗАмЕчАниЕ 3.4 . Если $g(t, x) \equiv$ const в $Q, \rho(t, x) \not \equiv$ const, но $\rho_{t}(t, x) \geqslant 0$ в $Q$, то условие (3.6) вьполнено всегда, а условие (3.7) будет вьполнено, например, при $T \geqslant T_{0}>0$, где $T_{0}$ явно вычисляется из условия (3.7). Таким образом, задача (1.1)-(1.4) будет иметь единственное решение, если высота цилиндра $Q \equiv Q_{T}$ достаточно велика.

4. Некоторые вспомогательные утверждения. Выше в работе мы неоднократно использовали возможность формального дифференцирования рассматриваемой задачи по переменной $t$. Для полноты изложения приведем подробное обоснование этого факта.

Итак, рассмотрим в $Q$ две первых краевых задачи

$$
\begin{aligned}
& \rho(t, x) u_{t}-\Delta u=h(t, x), \\
& u(0, x)=u_{0}(x), \quad x \in \Omega, \\
& u(t, x)=0, \quad t \in[0, T], \quad x \in \partial \Omega,
\end{aligned}
$$

и

$$
\begin{aligned}
& (\rho(t, x) w)_{t}-\Delta w=h_{t}(t, x), \\
& w(0, x)=\frac{\Delta u_{0}+h(0, x)}{\rho(0, x)}, \quad x \in \Omega, \\
& w(t, x)=0, \quad t \in[0, T], \quad x \in \partial \Omega .
\end{aligned}
$$


Заметим, что задача (4.4)-(4.6) получена формальным дифференцированием задачи (4.1)-(4.3) по $t$.

Предположим, что для функций $\rho(t, x), h(t, x), u_{0}(x)$ вьполнены условия $(\mathrm{A}),(\mathrm{C}),(\mathrm{D})$. В этом случае существует единственное энергетическое решение $u(t, x)$ (см. выше определение 2.2) задачи (4.1)-(4.3), которое в силу [7] принадлежит классу $W_{2}^{1,2}(Q)$.

Ниже нам понадобятся более слабые, нежели энергетические, решения задачи (4.4)-(4.6). Дадим соответствующее определение.

ОПРЕДЕЛЕНИЕ 4.1. Функция $w(t, x) \in L_{2}(Q)$ назьвается обобщенным $L_{2}$-решением задачи (4.4)-(4.6), если для любой $\chi(t, x) \in L_{2}\left(0, T ; \stackrel{\circ}{W}_{2}^{1}(\Omega)\right) \cap W_{2}^{1,2}(Q), \chi(T, x)=0$, выполнено интегральное тождество

$$
\begin{aligned}
& -\int_{\Omega} \rho(0, x) w_{0}(x) \chi(0, x) d x-\int_{Q} \rho(t, x) w \chi_{t}(t, x) d x d t-\int_{Q} w \Delta \chi(t, x) d x d t \\
& \quad=\int_{Q} h_{t}(t, x) \chi(t, x) d x d t
\end{aligned}
$$

где $w_{0}(x)=\left(\Delta u_{0}+h(0, x)\right) / \rho(0, x)$.

Справедлива

ТЕОремА 4.1. При выполнении условий $(\mathrm{A}),(\mathrm{C}),(\mathrm{D})$ функиия $u_{t}(t, x)($ әде $u(t, x)-$ решение задачи (4.1)-(4.3)) является энергетическим решением задачи (4.4)-(4.6).

ДокАЗАтЕльство. Покажем прежде всего, что обобщенное $L_{2}$-решение задачи (4.4)-(4.6) единственно.

Действительно, пусть существуют два обобщенных $L_{2}$-решения $w_{1}(t, x)$ и $w_{2}(t, x)$. Положим $y(t, x)=w_{1}(t, x)-w_{2}(t, x)$. Тогда $y(t, x)$ удовлетворяет интегральному тождеству

$$
\int_{Q} y(t, x)\left(\rho \chi_{t}+\Delta \chi\right) d x d t=0
$$

Рассмотрим в $Q$ задачу

$$
\begin{aligned}
& \rho(t, x) \chi_{t}-\Delta \chi=y(t, x), \\
& \chi(T, x)=0, \quad x \in \Omega, \\
& \chi(t, x)=0, \quad t \in[0, T], \quad x \in \partial \Omega .
\end{aligned}
$$

В силу [7] данная задача имеет единственное решение

$$
\chi^{*}(t, x) \in C\left(0, T ; \stackrel{\circ}{W}_{2}^{1}(\Omega)\right) \cap W_{2}^{1,2}(Q) .
$$

Положив в (4.7) $\chi(t, x)=\chi^{*}(t, x)$, мы, очевидно, получим, что $y(t, x) \equiv 0$ в $Q$.

Итак, обобщенное $L_{2}$-решение задачи (4.4)-(4.6) единственно. Покажем, что функция $u_{t}(t, x)$ (где $u(t, x)$ - решение задачи (4.1)-(4.3)) является обобщенным $L_{2}$-решением задачи (4.4)-(4.6).

Действительно, пусть $\chi(t, x) \in L_{2}\left(0, T ; \stackrel{\circ}{W}_{2}^{1}(\Omega)\right) \cap W_{2}^{1,2}(Q), \chi(T, x)=0$. Умножим уравнение $(4.1)$ на $-\chi_{t}(t, x)$ и проинтегрируем по прямоугольнику $Q$. После несложньх преобразований придем к соотношению

$$
-\int_{Q}\left(\rho u_{t}\right) \chi_{t} d x d t-\int_{Q} u_{t} \Delta \chi d x d t-\int_{\Omega}\left(\Delta u_{0}+h(0, x)\right) \chi(0, x) d x=\int_{Q} h_{t} \chi d x d t
$$


откуда вытекает, что $u_{t}(t, x)$ является обобщенным $L_{2}$-решением задачи $(4.4)-(4.6)$.

С другой стороны, в силу предположений $(\mathrm{A}),(\mathrm{C}),(\mathrm{D})$ задача (4.4)-(4.6) имеет энергетическое решение $w(t, x) \in C\left(0, T ; \stackrel{W}{2}_{2}^{1}(\Omega)\right)$, которое одновременно, очевидно, является и обобщенньм $L_{2}$-решением этой задачи. В силу доказанной вьше единственности такого решения для задачи (4.4)-(4.6) имеем $u_{t}(t, x) \equiv w(t, x)$, т.е. $u_{t}(t, x)$ является энергетическим решением задачи (4.4)-(4.6).

Теорема 4.1 доказана.

Автор благодарит профессора А.И. Прилепко и участников руководимого им семинара за полезное обсуждение результатов работы.

\section{СПИСОК ЦИТИРОВАННОЙ ЛИТЕРАТУРЫ}

[1] Прилепко А.И., Соловьев В.В. Теоремы разрешимости и метод Ротэ в обратных задачах для уравнения параболического типа // Дифференц. уравнения. 1987. Т. 23. № 11. C. $1971-1980$.

[2] Соловьев В.В. О разрешимости обратной задачи определения источника с переопределением на верхней крышке для параболического уравнения // Дифференц. уравнения. 1989. Т. 25. № 9. C. 1577-1583.

[3] Костин А. Б., Прилепко А. И. О разрешимости обратной задачи для уравнения теплопроводности // Обратные задачи для математических моделей физических процессов. Сб. научных трудов. М.: МИФИ, 1991. С. 52-58.

[4] Прилепко А.И., Костин А.Б. О некоторых обратных задачах для параболических уравнений с финальным и интегральным переопределением // Матем. сб. 1992. Т. 183. № 4. C. $49-68$.

[5] Ткаченко Д. С. Об одной обратной задаче для параболического уравнения с интегральным переопределением // Алгоритмический анализ неустойчивых задач. Тезисы докл. Всероссийской научн. конференции. Екатеринбург: Изд-во Уральского ун-та, 2001. С. 120.

[6] Ладыженская О. А., Солонников В. А., Уральцева Н. Н. Линейные и квазилинейные уравнения параболического типа. М.: Наука, 1967.

[7] Алхутов Ю. А., Мамедов И. Т. Первая краевая задача для недивергентных параболических уравнений второго порядка с разрывными коэффициентами // Матем. сб. 1986. Т. 173. № 12 . C. $477-500$.

[8] Ладыженская О. А. Математические вопросы динамики вязкой несжимаемой жидкости. М.: Наука, 1970.

[9] Кружков С.Н.Квазилинейные параболические уравнения и системы с двумя независимыми переменньми // Тр. семинара им. И. Г. Петровского. 1979. Т. 5. С. 217-272.

[10] Канторович Л.В., Акилов Г. П. Функциональный анализ в нормированных пространствах. М.: Физматгиз, 1959.

Московский государственный инженерно-физический институт

Поступило

E-mail: image@consultant.ru

06.08.2001 